\title{
Correlated Doping in Semiconductors: the Role of Donors in III-V Diluted Magnetic Semiconductors
}

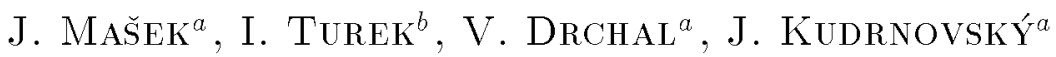 \\ AND F. MÁCA \\ ${ }^{a}$ Institute of Physics, AS CR, Na Slovance 2, 18221 Prague 8, Czech Republic \\ ${ }^{b}$ Institute of Physics of Materials, AS CR, Žižkova 22, 61662 Brno \\ and \\ Faculty of Mathematics and Physics, Charles University, Prague, Czech Republic

\begin{abstract}
We investigate the compositional dependence of the total energy of the mixed crystals (Ga,Mn)As co-doped with As, Sn, and $\mathrm{Zn}$. Using the ab initio linear muffin-tin orbital coherent potential approximation method we find a correlation between the incorporation of acceptors $(\mathrm{Mn}, \mathrm{Zn})$ and donors ( $\mathrm{Sn}$, antisite $\mathrm{As}$ ). In particular, the formation energy of $\mathrm{As}_{\mathrm{Ga}}$ is reduced by approximately $0.1 \mathrm{eV}$ in the presence of $\mathrm{Mn}$, and vice versa. This leads to the self-compensating behavior of $(\mathrm{Ga}, \mathrm{Mn}) \mathrm{As}$.
\end{abstract} \\ PACS numbers: 71.15.Ap, 71.20.Nr, 71.55.Eq, 75.50.Pp
}

\section{Introduction}

The Mn doping of the III-V semiconductors has two principal effects in the III-V diluted magnetic semiconductors (DMS). The correlated $d$-electrons at Mn atoms form local magnetic moments and the hybridization of the $d$-states with the band states results in various magnetoelectric and magnetooptical phenomena [1]. In addition, Mn atoms substituted for a trivalent cation act as acceptors and introduce holes into the valence band. It is now generally accepted [2] that the ferromagnetic coupling between the local moments in the III-V DMS is mediated by the mobile holes in the valence band.

In reality, however, the number of the holes is much smaller than the nominal concentration of $\mathrm{Mn}[3,4]$. This indicates that a large amount of compensating donors is present, with a strong effect on both conductivity and Curie temperature 
of these materials. As pointed out in [5], the nearly constant (or even decreasing) doping efficiency of order 0.1-0.2 can be explained only assuming that the number of the donors increases proportionally to the concentration of Mn. This was the reason to suggest that $\mathrm{Mn}$ atoms in the interstitial positions - being double donors - might have a principal role in the compensation [6].

Another favorite candidate for the compensating donor is an As antisite defect. These defects are well known in crystals grown with an excess As, in particular in the $(\mathrm{Ga}, \mathrm{Mn})$ As films [7]. So far, however, there was no reason to expect any correlation between the concentration of As antisites and the concentration of Mn.

Only recently, an increase in the number of the As antisites was indicated by comparing Curie temperature obtained from $a b$ initio calculations for (Ga,Mn,As)As mixed crystals [8] with experimental data. This finding opens a question whether and why the number of the As antisites is correlated with the level of Mn doping.

We investigate the correlation between the donors and acceptors in partly covalent III-V semiconductors such as GaAs. The cohesion energy of the covalent networks has a maximum if the Fermi energy lies within a band gap (Ioffe-Regel's rule [9]). Whenever the Fermi energy is situated in the valence or conduction band the strength of the bonds is reduced because the unfilled bonding states or occupied antibonding states appear, respectively. It is natural to expect that this mechanism, connected with the changes of the Fermi energy position in dependence on the compensation, leads to some kind of acceptor-donor correlation.

\section{Correlation energy}

We consider a crystal doped with both acceptors and donors. For simplicity, we assume that both acceptors and donors are substitutional impurities, which is the case of both As antisites and Mn in GaAs. Their concentrations are $x_{\mathrm{A}}$ and $x_{\mathrm{D}}$, respectively. The total energy of the doped crystal, normalized to a unit cell, is $W\left(x_{\mathrm{A}}, x_{\mathrm{D}}\right)$. We show first that the derivatives of $W\left(x_{\mathrm{A}}, x_{\mathrm{D}}\right)$ with respect to the concentrations $x_{\mathrm{A}}$ and $x_{\mathrm{D}}$ determine the formation energies of the defects, and also the correlation energy of the co-doping.

We start with a large unit cell (LUC) consisting of $N$ unit cells of the mixed crystal. The formation energy $E^{\mathrm{A}}$ of an acceptor $\mathrm{A}$ is defined as the reaction energy of the substitution process

$\mathrm{LUC}^{(\mathrm{X})}+\mathrm{A} \longrightarrow \mathrm{LUC}^{(\mathrm{A})}+\mathrm{X}$.

Here, $L U C^{(A)}$ is a large unit cell with one extra acceptor A replacing an atom $\mathrm{X}$ of the original $\mathrm{LUC}^{(\mathrm{X})}$. In our notation, the corresponding reaction energy is 


$$
E^{\mathrm{A}}\left(x_{\mathrm{A}}, x_{\mathrm{D}}\right)=N\left[W\left(x_{\mathrm{A}}+\frac{1}{N}, x_{\mathrm{D}}\right)-W\left(x_{\mathrm{A}}, x_{\mathrm{D}}\right)\right]+E_{\mathrm{atom}}(\mathrm{X})-E_{\mathrm{atom}}(\mathrm{A})
$$

The last two terms in Eq. (1) are the total energies of free-standing atoms X and A, respectively. The additional constant $E_{\text {atom }}(\mathrm{X})-E_{\text {atom }}(\mathrm{A})$, though crucial for the correct absolute value of the formation energy, does not depend on the actual composition of the material and so it is not important for the concentration-dependent effects we have in mind. With increasing size of the large unit cell, $N \rightarrow \infty$, the first term in Eq. (1) approaches the derivative of $W\left(x_{\mathrm{A}}, x_{\mathrm{D}}\right)$ with respect to $x_{\mathrm{A}}$. We have

$$
E^{\mathrm{A}}\left(x_{\mathrm{A}}, x_{\mathrm{D}}\right)=\frac{\partial W\left(x_{\mathrm{A}}, x_{\mathrm{D}}\right)}{\partial x_{\mathrm{A}}}+E_{\mathrm{atom}}(\mathrm{X})-E_{\text {atom }}(\mathrm{A})
$$

in a close analogy with the definition of the chemical potential. Similarly, the formation energy of a donor $\mathrm{D}$ substituting for an atom $\mathrm{Y}$ is

$$
E^{\mathrm{D}}\left(x_{\mathrm{A}}, x_{\mathrm{D}}\right)=\frac{\partial W\left(x_{\mathrm{A}}, x_{\mathrm{D}}\right)}{\partial x_{\mathrm{D}}}+E_{\mathrm{atom}}(\mathrm{Y})-E_{\mathrm{atom}}(\mathrm{D})
$$

Finally, the compositional dependence of the formation energies can be characterized by the correlation energy

$$
K\left(x_{\mathrm{A}}, x_{\mathrm{D}}\right)=\frac{\partial E^{\mathrm{A}}}{\partial x_{\mathrm{D}}}=\frac{\partial E^{\mathrm{D}}}{\partial x_{\mathrm{A}}}=\frac{\partial^{2} W\left(x_{\mathrm{A}}, x_{\mathrm{D}}\right)}{\partial x_{\mathrm{A}} \partial x_{\mathrm{D}}}
$$

The correlation energy can be alternatively, for finite large unit cells, expressed in terms of the four total energies corresponding to the reference $\mathrm{LUC}^{(\mathrm{XY})}$ and to related systems with an extra acceptor $\left(\mathrm{LUC}^{(\mathrm{AY})}\right)$, extra donor $\left(\mathrm{LUC}^{(\mathrm{XD})}\right)$, and both acceptor and donor ( $\left.\mathrm{LUC}^{(\mathrm{AD})}\right)$,

$$
K=W\left\{\mathrm{LUC}^{(\mathrm{AD})}\right\}-W\{\mathrm{LUC}(\mathrm{AY})\}-W\{\mathrm{LUC}(\mathrm{XD})\}+W\left\{\mathrm{LUC}^{(\mathrm{XY})}\right\} .
$$

The correlation energy $K\left(x_{\mathrm{A}}, x_{\mathrm{D}}\right)$ is positive if the formation energy of one impurity increases in the presence of the other. This means, in the case of semiconductors, that the material tends to be either $n$-type or $p$-type rather than a compensated semiconductor. On the other hand, negative correlation energy indicates that the presence of impurities of one kind makes the incorporation of the other dopants easier. In this case we can speak about a preferential compensation.

To investigate the correlation among the dopants, we use the coherent potential approximation (CPA) [10]. There are two reasons for this. First of all, CPA describes the configurationally averaged behavior of the mixed systems rather than particular arrangements of the impurities. This fits well to the thermodynamical Ioffe-Regel mechanism. In addition, the CPA is applicable to impure crystals with arbitrary concentrations of the dopants and it is particularly suitable for the description of the compositional dependence of the electronic properties. 


\section{Results}

We considered a series of multicomponent mixed crystals $\mathrm{Ga}_{1-x-y} \mathrm{~A}_{x} \mathrm{D}_{y} \mathrm{As}$ with acceptors $\mathrm{A}=\mathrm{Mn}, \mathrm{Zn}$ and donors $\mathrm{D}=\mathrm{As}$, Sn. The highly degenerate non-magnetic mixed crystals with $\mathrm{Zn}$ were studied in parallel to the diluted magnetic semiconductor $(\mathrm{Ga}, \mathrm{Mn})$ As to make clear that the acceptor-donor correlation does not depend on the presence of the magnetic moments. With our choice, all substitutions take place in the cationic sublattice. Only doping giving $p$-type materials was considered.

The $a b$ initio tight-binding linear muffin-tin orbital (TB-LMTO) version of the CPA [11] was used to calculate the electronic structure of the impure crystals and of their total energies. To obtain the derivatives of the total energy with respect to the concentrations, we varied both $x$ and $y$ on a fine mesh with $\delta x, \delta y=0.005$.

Table summarizes the calculated correlation energies $K(x, 0)$. The correlation energy of the co-doping is negative in all considered materials. The absolute value of $K$ is of order of a few electronvolts and it generally decreases with increasing level of the doping. The weakest correlation is found in $(\mathrm{Ga}, \mathrm{Mn}) \mathrm{As}$ compensated with Sn. The correlation energies for Sn are approximately twice smaller than the correlation energies for the As antisite, which is a double donor, as expected.

TABLE

Correlation energy for acceptors (A) and donors (D) in $p$-type mixed crystals $\mathrm{Ga}_{1-x} \mathrm{~A}_{x} \mathrm{As}$ at various levels of $p$-type doping.

\begin{tabular}{c|c|c|c}
\hline \hline$x$ & $\mathrm{~A}=\mathrm{Mn}, \mathrm{D}=\mathrm{As}$ & $\mathrm{A}=\mathrm{Zn}, \mathrm{D}=\mathrm{As}$ & $\mathrm{A}=\mathrm{Mn}, \mathrm{D}=\mathrm{Sn}$ \\
\hline 0.04 & $-3.36 \mathrm{eV}$ & $-5.48 \mathrm{eV}$ & $-1.39 \mathrm{eV}$ \\
0.05 & $-2.67 \mathrm{eV}$ & $-4.67 \mathrm{eV}$ & $-1.22 \mathrm{eV}$ \\
0.06 & $-2.21 \mathrm{eV}$ & $-4.19 \mathrm{eV}$ & $-1.09 \mathrm{eV}$
\end{tabular}

The negative correlation energy of the co-doping means that the formation energies of both acceptors and donors decrease in the presence of the compensating impurities. This is shown explicitly for the substitutional Mn and for the As antisite defect in Figs. 1 and 2. To avoid the technical problem with the additional constants in Eqs. (2), (3), we do not plot the entire formation energies $(\approx 2 \mathrm{eV})$, but only their changes with respect to the reference material, mostly $\mathrm{Ga}_{0.96} \mathrm{Mn}_{0.04} \mathrm{As}$.

Figure 1 shows how the formation energy of the As antisite defect changes with the concentration of $\mathrm{Mn}$. In addition to the main series of the data for $\mathrm{Ga}_{1-x} \mathrm{Mn}_{x}$ As we considered also a series of mixed crystals $\mathrm{Ga}_{0.99-x} \mathrm{Mn}_{x} \mathrm{As}_{0.01} \mathrm{As}$ already containing a small portion of the As antisites. In both cases, formation energy is reduced by approximately $0.1 \mathrm{eV}$ if the $\mathrm{Mn}$ concentration increases by a few atomic percent. 


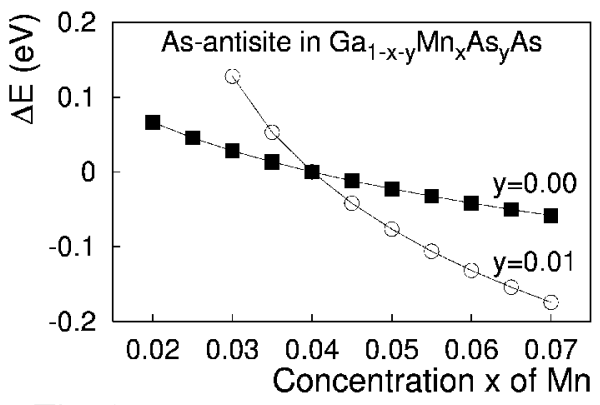

Fig. 1

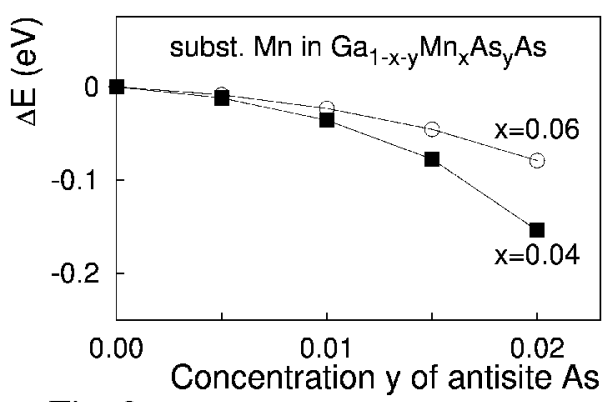

Fig. 2

Fig. 1. Dependence of the As antisite formation energy on the concentration $x$ of Mn acceptors for two series, $\mathrm{Ga}_{1-x} \mathrm{Mn}_{x} \mathrm{As}$ (boxes, $y=0.00$ ) and $\mathrm{Ga}_{0.99-x} \mathrm{Mn}_{x} \mathrm{As}_{0.01} \mathrm{As}$ (circles, $y=0.01$ ). The changes $\Delta E$ of the formation energy with respect to the reference systems with $x=0.04$ are shown.

Fig. 2. Change of formation energy, $\Delta E$, of substitutional $\mathrm{Mn}$ in Ga0.96-y $\mathrm{Mn}_{0.04} \mathrm{As}_{y} \mathrm{As}$ (boxes) and $\mathrm{Ga}_{0.94-y} \mathrm{Mn}_{0.06} \mathrm{As}_{y} \mathrm{As}$ (circles) due to the As antisite defects as a function of their concentration $y$.

The formation energy of the substitutional Mn was calculated for two series of mixed crystals, $\mathrm{Ga}_{0.96-y} \mathrm{Mn}_{0.04} \mathrm{As}_{y} \mathrm{As}$ and $\mathrm{Ga}_{0.94-y} \mathrm{Mn}_{0.06} \mathrm{As}_{y} \mathrm{As}$. Its variation with increasing amount of the As antisites is shown in Fig. 2. The changes of the formation energy are again of order of $0.1 \mathrm{eV}$.

To explain the results we turn to a simple model. In the one-electron picture, the total energy can be expressed in terms of the density of states $g(E)$,

$$
W=\int_{-\infty}^{E_{\mathrm{F}}} g(E)\left(E_{\mathrm{F}}-E\right) \mathrm{d} E
$$

The dependence of $W\left(x_{\mathrm{A}}, x_{\mathrm{D}}\right)$ on the chemical composition arises both from the redistribution of the electron states in the valence band due to the impurities and from the changes of the position of the Fermi level. In the case that $g\left(E_{\mathrm{F}}\right)$ does not change much with the chemical composition, the total energy depends on the concentrations of the impurities mostly via the position of the Fermi level. Because the extra acceptors (donors) push the Fermi level to lower (higher) energies, the correlation energy for the acceptor-donor co-doping is expected, according to Eqs. (5) and (6), to be always negative. Morever, as the variations of the $E_{\mathrm{F}}$ are inversely proportional to $g\left(E_{\mathrm{F}}\right)$, one can expect that also $W g\left(E_{\mathrm{F}}\right) \approx$ const. This is in a good agreement with the calculated decrease in the correlation energy with increasing concentration of Mn (cf. Table).

\section{Summary}

We showed that the formation energy of As antisite defects in GaAs decreases with increasing concentration of $\mathrm{Mn}$ or $\mathrm{Zn}$ in the cationic sublattice. The formation 
energy is reduced by approximately $0.1 \mathrm{eV}$ so that the number of these native defects can be largely enhanced in the presence of Mn. This effect may contribute to the self-compensation behavior of (Ga,Mn)As mixed crystals.

At the same time, the formation energy of the substitutional Mn is similarly reduced in the presence of the As antisites or by intentional co-doping with Sn. This can be interpreted that the presence of the donors, either native defects or intentional dopants, is important for an improved solubility of Mn in III-V materials.

Finally, the correlation between donors and acceptors seems to be a general feature tending to a self-compensation.

\section{Acknowledgment}

The financial support was provided by the Academy of Sciences of the Czech Republic (grant No. A1010214), by the Grant Agency of the Czech Republic (202/00/0122), and by RTN project "Computational Magnetoelectronics" of the European Commission (HPRN-CT-2000-00143)

\section{References}

[1] H. Ohno, J. Magn. Magn. Mater. 200, 110 (1999).

[2] T. Dietl, H. Ohno, F. Matsukura, J. Cibert, D. Ferrand, Science 287, 1019 (2000).

[3] B. Beschoten, P.A. Crowell, I. Malajovich, D.D. Awschalom, F. Matsukura, A. Shen, H. Ohno, Phys. Rev. Lett. 83, 3073 (1999).

[4] K.W. Edmonds, K.Y. Wang, R.P. Campion, A.C. Neumann, C.T. Foxon, B.L. Gallagher, P.C. Main, cond-mat/0205517.

[5] F. Máca, J. Mašek, Phys. Rev. B 65, 235209 (2002).

[6] J. Mašek, F. Máca, Acta Phys. Pol. A 100, 319 (2001).

[7] H. Shimizu, T. Hayashi, T. Nishinaga, M. Tanaka, Appl. Phys. Lett. 74, 398 (1999).

[8] J. Kudrnovský, I. Turek, V. Drchal, F. Máca, J. Mašek, P. Weinberger, submitted to Phys. Rev. B.

[9] A.I. Ioffe, A.R. Regel, Prog. Semicond. 4, 237 (1960).

[10] B. Velický, S. Kirkpatrick, H. Ehrenreich, Phys. Rev. 175, 747 (1968).

[11] I. Turek, V. Drchal, J. Kudrnovský, M. Šob, P. Weinberger, Electronic Structure of Disordered Alloys, Surfaces and Interfaces, Kluwer, Boston 1997. 